\title{
Neurovascular 4DFlow MRI (Phase Contrast MRA): emerging clinical applications
}

\author{
Patrick Turski ${ }^{*}$, Andrew Scarano ${ }^{1}$, Eric Hartman ${ }^{1}$, Zachary Clark', Tilman Schubert ${ }^{3}$, Leonardo Rivera ${ }^{2}$, Yijing Wu ${ }^{2}$, \\ Oliver Wieben ${ }^{2}$ and Kevin Johnson ${ }^{2}$
}

\begin{abstract}
Recent advances in 4DFlow MRI (Phase Contrast MRA) acquisition and reconstruction enable high resolution exams to be obtained in practical imaging times. 4DFlow MRI provides images of vascular morphology and quantitative measurements of blood velocity throughout a 3D imaging volume. Hemodynamic parameters such as flow volume, relative wall shear stress, streamlines, vorticity and pressure gradients can be derived from the velocity data. The combination of anatomic vessel wall imaging, lumen visualization and physiologic data derived from accelerated 4DFlow MRI augments the characterization of intracranial arterial stenosis, aneurysms, vascular malformations and dural sinus pathology. This review provides an update for clinicians interested in 4DFlow MRI of the brain.
\end{abstract}

\section{Background}

Blood flow is altered in a variety of diseases; however, clinically viable methods for evaluating intracranial arterial and venous flow have been limited. With recent advances, blood velocities can be measured with transcranial Doppler ultrasound, magnetic resonance imaging (MRI) and potentially by digital subtraction angiography (DSA). Of these modalities, MRI is an appealing imaging option due to its non-invasive nature, ability to image vessels with complex geometry, high spatial resolution and ability to correlate well with other imaging features. The ability to measure velocities by flow encoded MRI was demonstrated by Moran [1] in 1983, shortly after MR was implemented for clinical imaging. Using this strategy flow encoding was implemented for 2D and 3D acquisition by Dumoulin [2, 3] and clinical applications for vascular flow imaging were expanded by Pelc et al [4] and Wigstrom et al [5]. However, general use of the flow imaging was limited by long acquisition times, complex implementation and motion artifacts. Consequently, for several decades flow measurements with MRI played only a minor role in neurovascular assessment.

Recently, substantial technology has been developed which has dramatically expanded the opportunities for

\footnotetext{
* Correspondence: pturski@uwhealth.org

${ }^{1}$ Department of Radiology, University of Wisconsin School of Medicine and

Public Health, Madison, Wisconsin, USA

Full list of author information is available at the end of the article
}

flow assessment with MRI. These include faster, stronger and more stable imaging gradients, phased array receiver technology and orders of magnitude increases in reconstruction hardware. Together and as detailed in this article, these technologies have allowed for cardiac cycle time resolved, 3D flow imaging (4DFlow) to be obtained with clinically relevant resolution in relatively short imaging times. 4DFlow acquires a volumetric imaging acquisition which allows the retrospectively visualization and quantification of complex flow fields. Recent interest from the MRI and post-processing software vendors along with promising clinical studies suggest that 4DFlow MRI will likely become widely available in the near future.

Clinical applications of 4DFlow MRI are now expanding and 4DFlow is increasing used to supplement MRI and MRA exams. The ability to quantitatively measure velocity has many applications in neurovascular imaging [6]. However, clinical implementation of 4DFlow MRI requires an understanding of the strengths and limitations of the technique. This overview discusses the basic features of 4DFlow MRI from a clinical perspective. The review outlines the role of 4DFlow MRI in the detection and characterization of arterial stenosis, atherosclerotic disease, aneurysms, vascular malformations and dural sinus pathology. 


\section{Intracranial 4DFlow MRI acquisition}

The principles for flow measurement remain similar to those initially proposed by Moran [1]. Briefly, when a spin is moving along the magnetic field gradient its resonance frequency changes whereas for a static spin the resonance frequency would remain constant. Thus, if a gradient is applied for some time and an equal and opposite gradient is applied for the same time (flow encoding gradients) static spins will rotate faster and then slower such that the net change in frequency (rotation speed) is zero. For moving spins, the faster and slow frequency will not cancel. For each spin, this results in a phase shift in the acquired signal which is directly proportional to velocity. If the flow encoding gradients are small enough and the motion is coherent (e.g. blood flow) the phase difference in the detected signal is directly proportional to the average velocity in the voxel of interest. This phase difference is only detectable up to a $\pm 180^{\circ}$ in each direction and the minimum velocity that causes a $180^{\circ}$ rotation is the velocity encoding, $V_{\text {enc. }}$ Due to this fixed dynamic range, to avoid aliasing, the $\mathrm{V}_{\text {enc }}$ must be selected to be only slightly higher than the maximum expected velocity. As demonstrated in Fig. 1, for angiograms generated from a 4D flow acquisition, there is a tradeoff between the sensitivity to slow flow and the range of detectable velocities. In some instances, such as in the case of imaging venous flow, the velocity encoding is selected to maximize the signal from slow flow and aliasing of higher velocities is identified and accepted in the images as signal loss.

4DFlow MRI is based on an RF and gradient spoiled 3D gradient echo sequence (i.e. SPGR/FLASH/TFE/Fast FE). Starting from a standard sequence, flow encoding

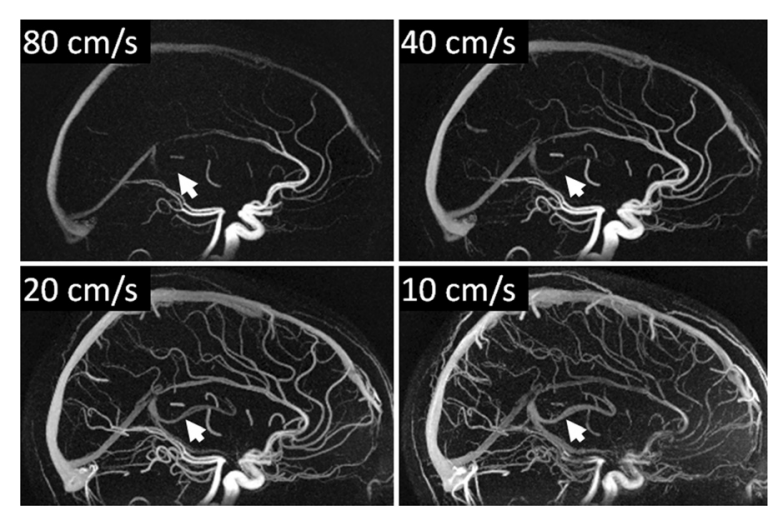

Fig. 1 Limited maximum intensity projections (MIPS) of angiograms generated from 4DFlow acquisitions with $V_{\text {enc }}$ 's of 80, 40, 20 and $10 \mathrm{~cm} / \mathrm{s}$. As the $V_{\text {enc }}$ is reduced to $10 \mathrm{~cm} / \mathrm{sec}$ the visualization of large arterial vessels is reduced. In these vessels, the measured velocity values will be unreliable due to limited detectable velocity dynamic range. At the same time, the visualization of slow flowing venous structures is improved as the $V_{\text {enc }}$ is reduced gradients are inserted after excitation and before the imaging readout, as shown in Fig. 2. This increases the echo time (TE) and repetition time (TR) by $\sim 1-3 \mathrm{~ms}$ depending on the $\mathrm{V}_{\text {enc }}$ and scanner gradient performance. Further, in order to measure flow in 3 directions at least 4 images must be collected with different flow encoding gradient directions, 1 for background phase and 1 for each direction. Typical implementations interleave these encodings. Finally, cardiac gating is incorporated either though prospective or retrospective triggering. For intracranial applications typically 10-20 cardiac phases are acquired. This results in cardiac time resolved 3D volumes, which are subtracted to produce flow fields (Fig. 3). Considering the increase in TR, the need for at least 4 flow encodings, and a minimum 10 cardiac phases, the acquisition time for a $4 \mathrm{D}$ flow exam is $\sim 50$ 100x longer than a traditional gradient echo scan, such as those used for contrast enhanced angiography.

Fortunately, technologic advances have shorted imaging time and increased spatial and temporal resolution. Current implementation varies from site to site and vendor to vendor but follows similar trends. Nearly all implementation of 4DFlow utilize parallel imaging, most commonly direct techniques such as SENSE or GRAPPA [7-9] but also with indirect methods such as using localized coils. In the case of Cartesian imaging, this reduces the required scan time by a factor of 2-4; and is typically not sufficient to reduce scan times for clinically relevant coverage and spatial resolution $(<1 \mathrm{~mm})$ to feasible scan times. One potential method for reducing scan times is to use non-Cartesian sampling trajectories which either collect more data per excitation, as in spiral [10] or allow greater under sampling of the imaging volume without obscuring artifacts, as in radial trajectories. Non-Cartesian imaging additionally allows for a reduction of the echo time $[11,12]$, allowing improved imaging of complex flow and/or higher spatial resolution $[13,14]$. Another method commonly used to reduce scan time is to utilize a reconstruction which harnesses correlation in space and time [9, 15-22]. These techniques make the observation that most signal is from non-moving tissues and thus the same in all the time frames. The amount of the acceleration possible with these techniques is extremely high but requires substantial computational power to fully achieve high levels of acceleration [23]. More modest accelerations can be achieved utilizing lower computational burden of $\mathrm{k}-\mathrm{t}$ acceleration techniques [19]. With the combination of parallel imaging and $\mathrm{k}-\mathrm{t}$ acceleration techniques, the major arterial circulation can be imaged with $1 \mathrm{~mm}$ isotropic spatial resolution within 5-10 $\mathrm{min}$. The joint combination of parallel imaging, spatiotemporal acceleration and non-Cartesian sampling provides the opportunity to further increase spatial resolution $(<0.7$ isotropic) and provide whole brain coverage [14]. While vendors' 


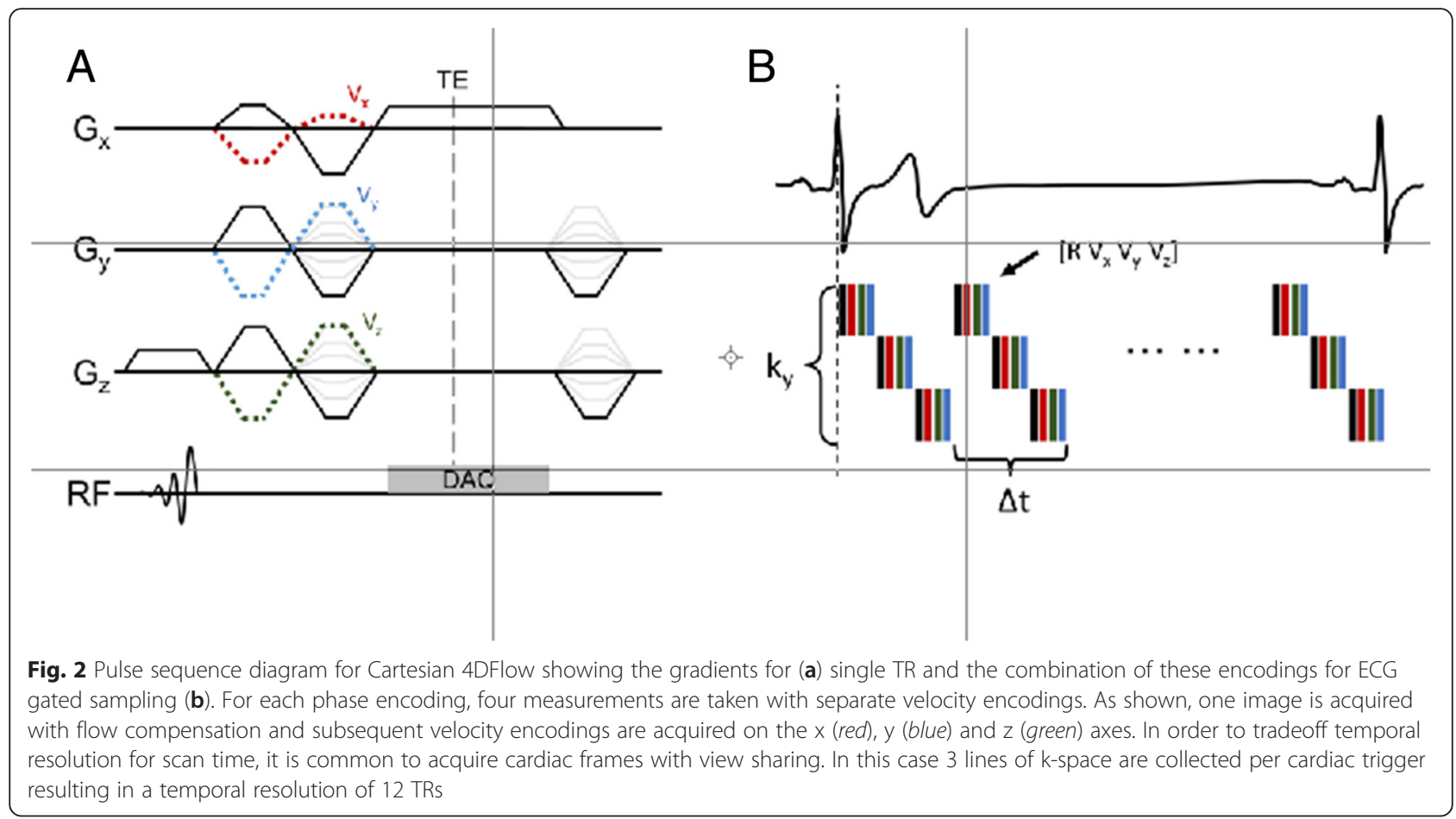

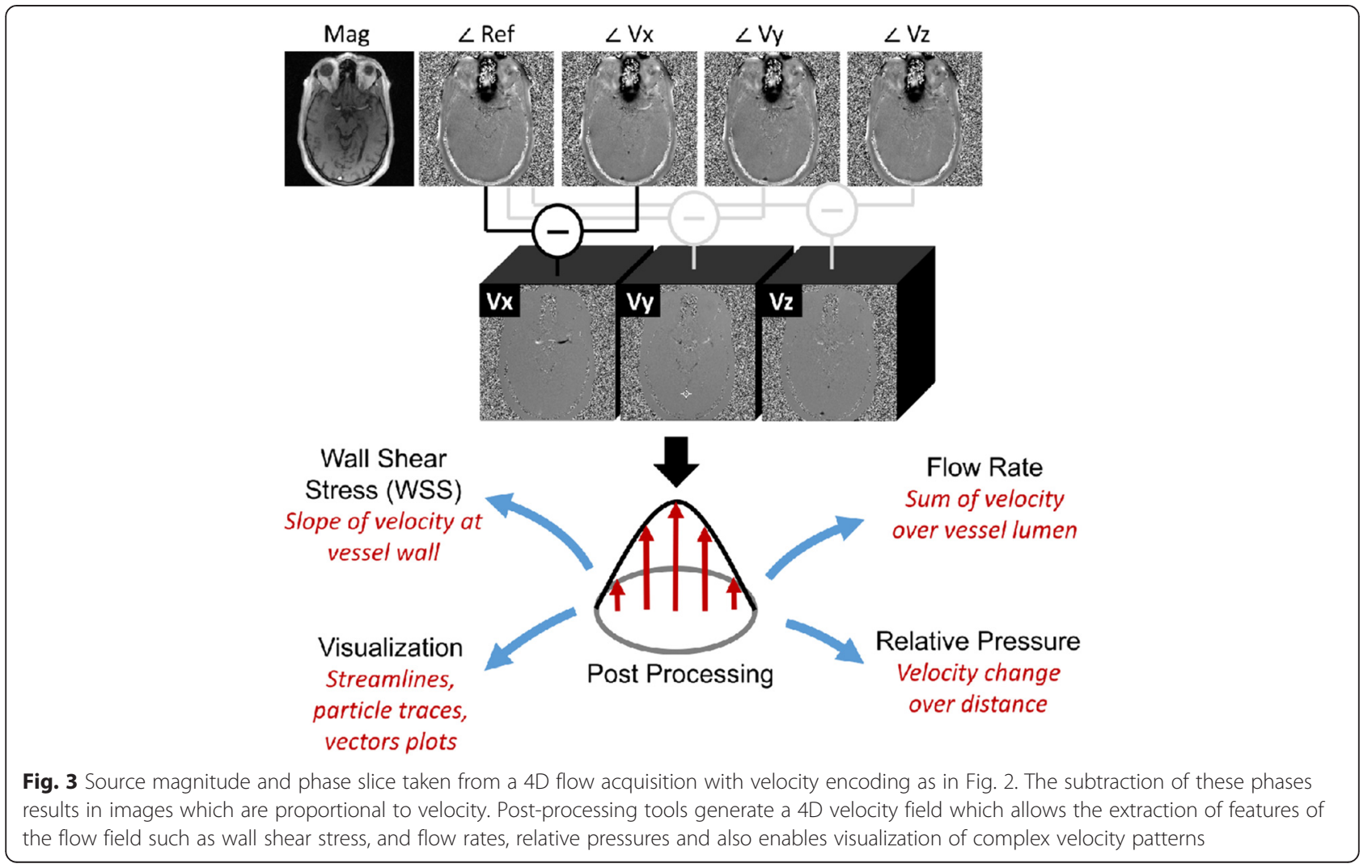


have not released specific 4D flow packages, it is most likely that products will be based on simple $\mathrm{k}-\mathrm{t}$ acceleration, Cartesian sampling and parallel imaging; with computational hurdles existing for advanced constrained reconstructions and non-Cartesian approaches.

\section{DFlow MRI display and flow analysis}

4DFlow imaging acquisitions produce a large number of images which can be challenging to interrogate with standard imaging viewing and analysis platforms. In scenarios where advanced processing is unavailable, there are several components of the 4DFlow MRI data readily available for clinical assessment. The magnitude images display both stationary tissue and flow compensated vascular structures providing the opportunity to precisely related soft tissue abnormalities to the adjacent vessels. Following subtraction of the stationary background tissues, the flow (or speed) images are displayed without specifying the direction of flow. An important feature is that the signal intensity of the flow images is dependent on the velocity encoding selected at the time of the acquisition; flow velocities that closely approximate the velocity encoding will have the highest signal intensity.

Alternatively, each flow encoding direction can be independently displayed. In this instance, the velocity images are reconstructed with velocity displayed in the superior/inferior, anterior/posterior and right/left using a gray scale or color display. Velocity is a vector that has direction and magnitude, if a color display is used, direction is displayed by the chosen color scheme and magnitude is displayed as brightness. Velocity aliasing occurs when the velocities exceed the velocity encoding. In this scenario the pixel signal intensity will flip to display flow in the opposite direction. If a gray scale display is used the pixels change from bright to dark when aliasing occurs incorrectly indicating the direction of velocity and flow.

A more complete and quantitative interrogation of the 4DFlow data is possible with dedicated flow analysis software. This allows the simultaneous visualization of the direction and magnitude of the velocity field and can enable flow measurements of specific vessels. The initial step in flow analysis is the segmentation of the vascular structures from the imaging volume. The resulting vascular and flow data are subsequently analyzed using a 4DFlow package. To date, numerous packages have been adopted most frequently these have been tools developed for generalized visualization of flow fields (e.g. EnSight; CEI, Apex, NC, applications in the aerospace industry); however, FDA approved tools are now available (e.g. Arterys, San Francisco, CA USA). Such flow analysis packages provide software tools allowing for navigation throughout the 3D flow field. The flow field can be transected by 2D planes at the desired location and angle. Velocity and flow rate (flow rate = velocity $\mathrm{x}$ cross sectional area of the vessel) can be measured from a 2D plane orthogonal to the vessel of interest. The flow field can also be displayed as color coded velocity vectors representing the magnitude and direction of flow. Streamline displays are virtual instantaneous paths that are tangent to the velocity vectors at the specified point in time. Streamlines are relatively easy to interpret and provide an intuitive sense of the hemodynamics. Particle paths are another option and represent virtual particles that follow the velocity vectors from one time point to another simulating the flow of particles in the flow channels. Many important derivatives of velocity can be calculated. Estimations of wall shear stress can be generated by calculating the velocity gradient perpendicular to the vessel wall, WSS can be thought of as the drag of the flowing blood on the endothelial surface [24]. Measurements of pulsatility can be obtained and expressed as the pulsatility index $(\mathrm{PI}=(\mathrm{QmaxQmin}) / \mathrm{Qmean} ; \mathrm{Q}=$ flow $\mathrm{mL} / \mathrm{min}$ ) [25]. Vorticity is a curl in the velocity field and measures local rotation, vorticity can be quantified and has the potential to improve characterization of aneurysm flow [26]. Relative pressure variations within the velocity field can be calculated by using the Pressure Poisson Equation derived from the Navier-Stokes equations. For example, relative pressure drop across a stenosis can be determined from the velocity measurements using this approach [27] (Fig. 3). All of these measurements are limited by partial volume effect, phase errors, aliasing and motion. Fortunately, strategies are in development to speed analysis by using automated segmentation, quantification, aliasing corrections and methods to improve streamline, and particle path displays by reducing vector divergence [28-32].

\section{Clinical applications 4DFlow MRI of intracranial atherosclerosis}

The current standard of care for treatment of symptomatic intracranial atherosclerotic disease is medical therapy rather than percutaneous endovascular therapy due in large part to the SAMMPRIS trial in 2011 [33]. However, the only imaging parameter utilized in this trial was percent vessel stenosis. Furthermore, about $12 \%$ of the medical therapy group reached the primary endpoint of stroke or death within 1 year of a symptomatic stroke related to an atherosclerotic plaque raising the likelihood that a certain subset of patients could benefit from more advanced risk stratification and treatment options.

Certain hemodynamic conditions predispose patients to the development and progression of atherosclerotic plaques. The most clearly identified relationship of this nature is the development of atherosclerotic disease in regions of low wall shear stress. Wall shear stress (WSS) is detected by the endothelial cell surface mechanoreceptors 
which signal the cell regarding the presence of elevated, normal or low wall shear stress. Normal or high wall shear stress promotes reorganization of the cell cytoskeleton, tightens endothelial cell junctions and reduced the endothelial cell permeability to lipids. Whereas, low wall shear stress promotes endothelial cell disorganization, reduces tight junctions and increases permeability to lipids [34]. For example, atherosclerotic disease is most often encountered in the bulb of the carotid bifurcation where there is recirculating flow and low wall shear stress demonstrated by 4Dflow MRI [35-37].

Studies of intracranial stenosis hemodynamics using computational fluid dynamic models (CFD) based on 3D CTA data have been performed in patients with stenosis of the proximal middle cerebral artery greater than $70 \%$. In a study of 32 patients, the risk of recurrent stroke increased as the spatial gradient of the flow velocity increased within the stenosis. Interestingly, the risk of recurrent stroke did not correlate with the degree of stenosis measured by DSA in this group of patients [38].

Intracranial arterial WSS has also been studied with 4Dflow MRI. Large vessel arterial wall shear stress decreases with age, drops significantly during the 5th decade of life, is lower in males than females, and is lowest in the anterior cerebral arteries [39]. These relationships are likely influenced by multiple factors as humans age including increased vessel diameter, decreased blood flow [39], and other contributions such as cardiac output, blood pressure and hormone effects. Other 4DFlow MRI studies have been used to assess vertebrobasilar insufficiency [40], endovascular stenting [41], subclavian steal [42], extracranial-intracranial (EC-IC) bypass [43], and cerebrovascular blood flow reactivity to carbon dioxide inhalation [44].

Recent reports using contrast enhanced 3D T1 weighted black blood arterial wall imaging have demonstrated that intracranial atherosclerotic plaque wall enhancement is associated with an increased likelihood that the plaque is the culprit plaque responsible for the ischemic event $[45,46]$. The wall enhancement has been shown to decrease with time after the event [47]. Intracranial plaque morphology including intra-plaque hemorrhage $[48,49]$ and increased lipid rich necrotic core volume [50] have also been established as stroke risk factors. In addition, carotid artery studies have demonstrated that complex plaque morphology including intra-plaque hemorrhage, thrombus or fibrous cap rupture is located predominantly on the ipsilateral side of stroke [51-53].

4DFlow MRI provides an opportunity to explore wallflow-lesion interactions utilizing the combination of vessel wall imaging, 4Dflow and vessel lumen morphology. Unfortunately, achieving the high spatial resolution required to quantitatively depict the intracranial vasculature is challenging due to competing factors including SNR, scan time and artifacts. This is especially true for 4Dflow and CE-MRA. Current investigations of ultrashort echo (UTE) imaging suggest that UTE increases acquisition efficiency while offering improved flow compensation, increased spatial resolution and robustness to artifacts [14] (Fig. 4).

\section{DFlow MRI of intracranial aneurysms}

There is intense investigation into aneurysm hemodynamics and how flow contributes to aneurysm formation, growth and rupture. Although the major of investigations have been based on computation fluid dynamics modeling (CFD) [54-58], it is clear that 4DFlow MRI provides information similar to CFD and can contribute to the hemodynamic assessment of intracranial aneurysms [24, 59-62].

Aneurysm hemodynamic patterns can be grouped into three categories: 1) entry events, 2) intrasaccular flow characteristics and 3) wall/flow interactions. Entry events

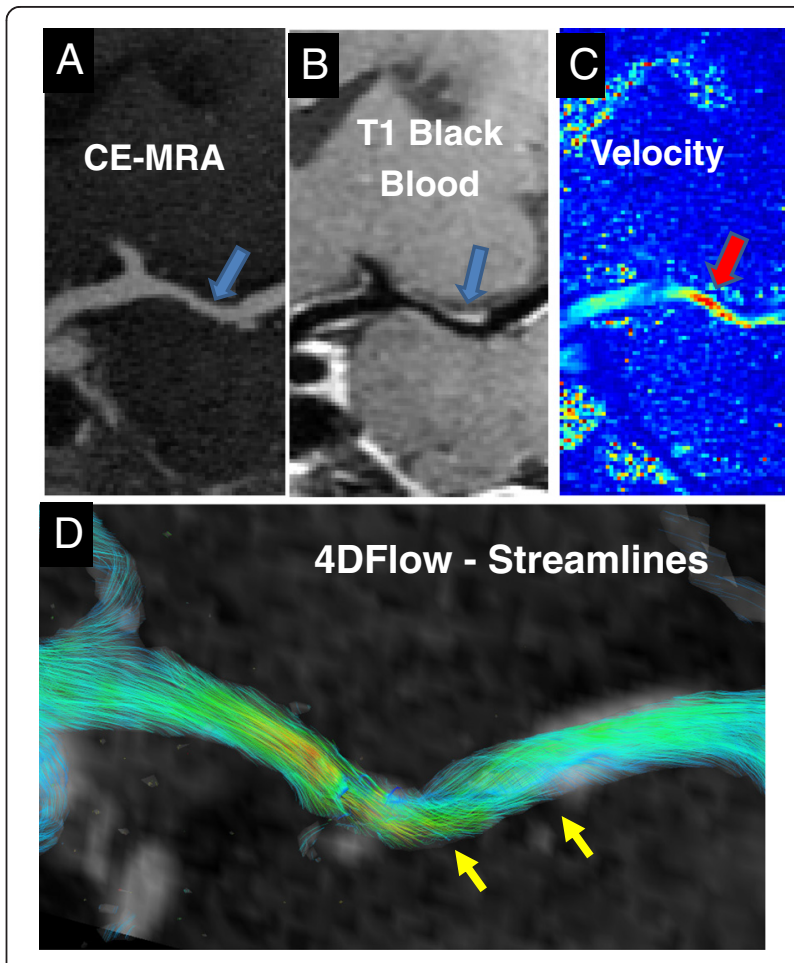

Fig. 4 Left middle cerebral artery stenosis in a patient with diffuse atherosclerotic disease. Images a-c are displayed as curved vessel reformations. a The ultra short echo time CE MRA demonstrates a stenosis of the proximal left middle cerebral artery (arrow). b Arterial wall enhancement is seen on post contrast T1BB at the location of the stenosis (arrow) and distal to the stenosis. c Elevated 4Dflow MRI velocities derived from the ultra short echo time acquisition are identified within the stenosis suggesting that the lesion is hemodynamically significant. $\mathbf{d}$ Streamlines generated through the stenosis demonstrate a helical flow pattern (arrows) 
are comprised of measured inflow concentration indices related to the inflow jet. Intrasaccular flow characteristics consider features such as vorticity, vortex core line length as well as ratios of kinetic energy, shear strain rate and viscous dissipation. Finally, wall/flow interactions include maximum and minimum WSS measurements, shear concentration index, area of aneurysm exposed to low WSS, and oscillatory shear index [63].

Sforza et al [63] also found that the hemodynamic environment predisposing to aneurysm growth was composed of a concentrated inflow stream leading to complex intrasaccular flow patterns; specifically, patterns characterized by areas of concentrated high WSS accompanied by larger areas of low WSS. They concluded that the concentrated inflow stream disperses into complex intravascular flow streams that produce non-uniform WSS distributions with concentrated areas of high WSS and large areas of low WSS. They propose that these hemodynamic conditions represent the features which promote aneurysm growth. They also noted that the same trends were observed in rupture versus non ruptured aneurysms. As 4DFlow MRI methods evolve, similar measurements of in vivo hemodynamics will be possible and will be able to provide objective criteria to aid in aneurysm risk stratification.

Meng et al. [64] tried to unify conflicting CFD data by proposing that in large aneurysms low WSS promotes wall inflammation through an inflammatory cell mediated pathway leading growth. Whereas, in small aneurysms or blebs high WSS and a positive WSS gradient promotes a different mural cell mediated inflammation pathway leading to growth and rupture.

Using contrast enhanced 3D T1 black blood wall imaging, several reports have demonstrated an association of aneurysm wall enhancement with aneurysm growth or rupture [65-67]. Additional approaches are being developed that combine 4DFlow MRI and 3D T1 black blood imaging of the aneurysm wall to link aneurysm wall thickness and wall enhancement to specific flow dynamics [68] (Fig. 5).

To provide risk stratification, the flow and wall imaging features will need to be correlated with other known vascular health factors (smoking, hypertension, family history, collagen vascular disease) and individual patient details (size, location, age, gender) to allow further predictability for risk of aneurysm growth and rupture [69].

\section{DFlow vascular malformations}

Imaging cerebrovascular arteriovenous malformations (AVMs) with high flow and rapid arteriovenous shunting presents several challenges. The malformations are often supplied by multiple arterial pedicles and have complex anomalous venous drainage necessitating a large field of view that encompasses the majority of the cerebrovascular system. High spatial resolution for imaging small vessels and high temporal resolution for detecting rapid arteriovenous shunting is needed to adequately characterize AVMs. The classification of AVMs requires description of size, location, arterial supply and venous drainage. This
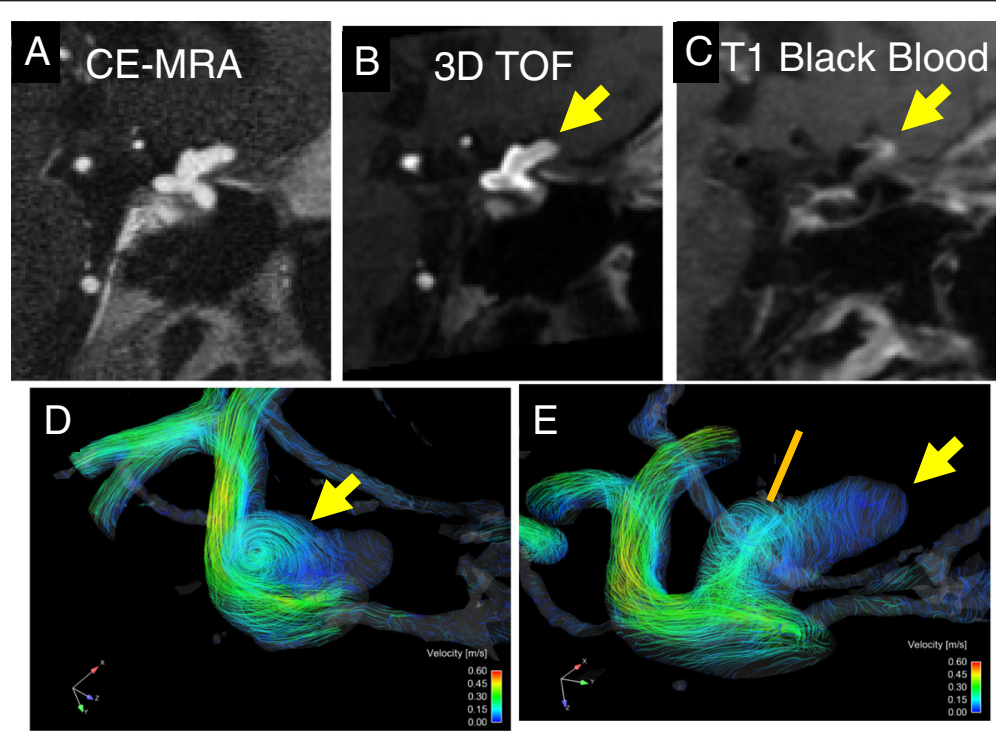

Fig. 5 Aneurysm of the supraclinoid carotid artery. $\mathbf{a}$ and $\mathbf{b}$ The CE MRA and 3D TOF MRA demonstrate that the aneurysm has a more spherical proximal component and a distal outpouching (arrow). c The distal outpouching and apex of the aneurysm enhance on the 3D T1 weighted contrast enhanced black blood image (arrow). $\mathbf{d}$ and $\mathbf{e}$ The streamline displays reveals vortex flow (arrow) and a central vortex core (line) in the proximal spherical component of the aneurysm. The distal outpouching has low flow velocity and presumed low wall shear stress 
information enables Spetzler-Martin grading [70] which predicts operative morbidity and mortality. DSA has long been considered the gold standard for full characterization of AVMs due to exceptional temporal and spatial resolution. However 4DFlow MRI has emerged as a tool capable of characterizing size, location, arterial supply and drainage noninvasively. A major advantage of 4DFlow MRI is the ability to provide additional physiologic information including velocity, flow volume, wall shear stress, pressure gradients, streamlines and flow path lines [71]. The hemodynamic features combined with the morphological image provide an expanded perspective on the impact of the AVM on global cerebrovascular blood flow (Fig. 6). Selective vessel imaging can be accomplished by vessel segmentation and seeding of streamlines at the origin of the vessels of interest [31, 72] (Fig. 7).

Wu et al [73] demonstrated that there was a relationship between the macrovascular hemodynamics of AVMs and the microvascular effects on tissue perfusion. In this study, higher grade Spetzler-Martin AVMs were associated with higher peak velocity and blood flow in feeding arterial vessels and higher blood flow in draining veins. As postulated previously, these macrovascular parameters lead to a steal phenomenon affecting microvascular tissue perfusion with an inverse relationship between perinidal perfusion measures including cerebral blood flow (CBF) and cerebral blood volume (CBV) with macrovascular arterial and venous blood flow. However, these parameters did not predict symptomatic presentation and were not associated with other previously described AVM risk factors (deep AVM location, deep venous drainage, associated aneurysm or venous stenosis). Time integrated 3D path lines of the entire cerebrovasculature can be color coded to provide a pre surgical roadmap of large feeding arteries and draining vessels. This approach has been successfully used to select target vessels for staged embolization and to follow the results of embolization [74]. Recent work by Wu et al [75] also demonstrates the value of 4DFlow MRI to precisely define the hemodynamics of a particularly complex AVM guiding surgical intervention.

4DFlow MR Imaging has also proved useful to assess dural arteriovenous fistulas (DAVFs). Delineation of the arterial anatomy and venous drainage is essential for the Cognard classification [76] of DAVFs. Similar to AVMs the 4DFlow data can be used to isolate flow in individual vessels providing selective display of streamline flow. Using this technique it may be possible to classify DAVFs using the Cognard system and provide a vascular roadmap for surgical planning $[29,72]$.

In contrast to AVMs, DAVFs often present less aggressively with symptoms of pulsatile tinnitus rather than intracranial hemorrhage. Often times the only imaging clue to the presence of a DAVF is asymmetric early venous filling on time resolved contrast enhanced MRA. A new approach called HYPRFlow combines a dynamic
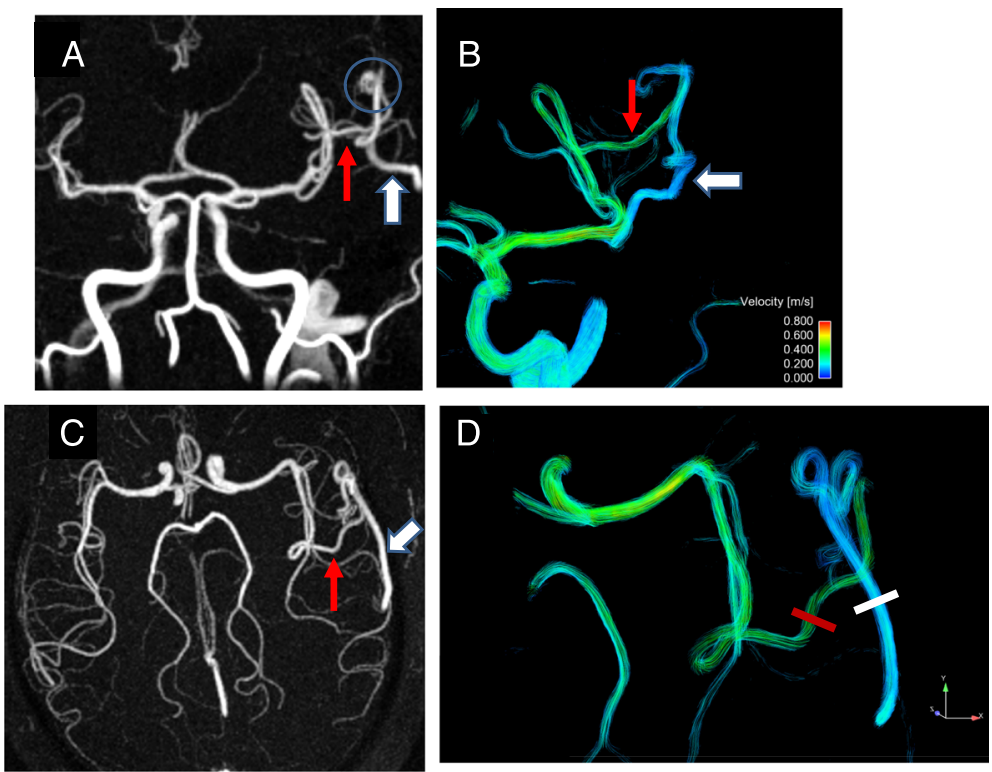

Fig. 6 Left frontal lobe Arteriovenous Malformation. a 4D Flow Coronal MIP demonstrates an enlarged Left MCA opercular branch (red arrow) supplying a small frontal lobe AVM (circle) and a cortical draining vein (white arrow). b Streamline flow displayed in the coronal plane reveals preferential flow in the feeding artery (red arrow) and draining cortical vein (white arrow). c 4DFlow Axial MIP displaying the main arterial feeder (red arrow) and largest draining cortical vein (white arrow). $\mathbf{d}$ Quantitative 4D flow analysis (axial projection) reveals moderately elevated flow volume in the arterial feeder of $55 \mathrm{~mL} / \mathrm{min}$ (measured at the red lined) and nearly matching venous outflow of $52 \mathrm{~mL} / \mathrm{min}$ (measured at the blue line) 

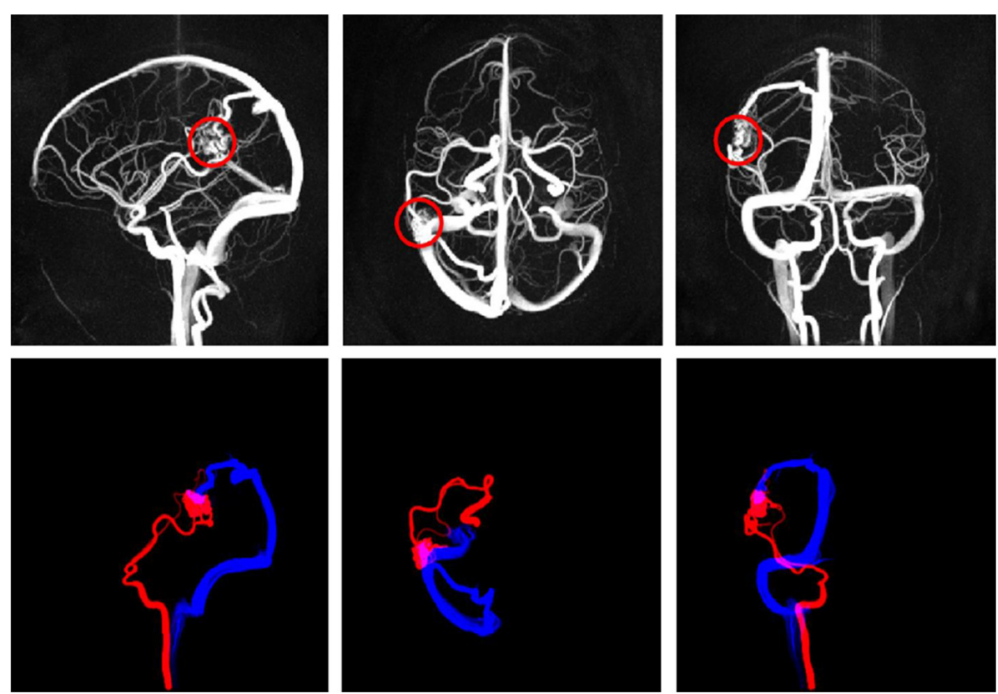

Fig. 7 4DFlow MRI Cartography of a right parietal brain AVM. Top row: Sagittal, axial and coronal 4DFlow MRI MIP images of the AVM. Bottom row: Selective streamline display demonstrates a large MCA branch feeding artery and the dominant draining cortical vein. Images courtesy of Michael Loecher, PhD

time resolved contrast enhanced MRA during the passage of a contrast bolus with a 4DFlow acquisition which is used to improve the image quality of the time series using an innovative reconstruction method (constrained reconstruction) [77]. Initial experience using HYPRFlow MRI with DAVFs demonstrated excellent arteriovenous separation, high resolution vascular images and concordant Cognard classification with DSA [78].

\section{DFLOW MRI dural sinuses}

4DFlow MRI is the only imaging modality that can assess global intracranial venous blood flow and hemodynamics in adults. 4DFlow MRI has been shown to be accurate and reproducible for measuring dural sinus flow velocity, flow rate and demonstrating streamline flow in normal subjects [79]. In the sagittal sinus, blood flow velocity and blood flow rate increases from anterior to posterior as the sagittal sinus increases in size. The transvers sinus velocities and flow rates are dependent on the anatomic configuration of the transverse sinus and torcular herophili. Small venous structures are less reliably assessed due to limitations in spatial resolution and low signal due to very slow flow. Reference values for dural sinus velocity and blood flow rates in normal subjects are available for comparison across age groups and provide a foundation for developing clinical applications [79].

The role of 4DFlow MRI in the diagnosis and follow up of dural sinus thrombosis continues to evolve. Recent comparisons of 4DFlow MRI to contrast enhanced 3D GE T1 weighted imaging demonstrated false positive results for 4DFlow MRI ranging from 4.7 to $8.2 \%$. This was due to signal loss in regions of complex/turbulent flow, susceptibility artifacts and very slow flow. 4DFlow
MRI also missed $10.4 \%$ of thrombosed segments detected by 3D GE T1 weighted imaging [80, 81]. One limitation of this comparison is that the authors did not include the magnitude images in the review. In the setting of possible dural sinus thrombosis, the 4DFlow MRI exam should be obtained following the administration of a gadolinium contrast agent. In this instance, the magnitude images from the 4DFlow MRI acquisition are the equivalent of contrast enhanced 3D GE T1 weighted images. The contrast enhanced magnitude images are not flow encoded and thus do not suffer from velocity dependent signal loss. Including these images would likely have reduced the number of false positives. In addition to the magnitude images, 4DFlow provides objective blood flow data that allows for qualitative and quantitative assessment of treatment related improvements in dural sinus blood flow [79].

Another important application of 4DFlow MRI is the assessment of dural sinus flow in patients with intracranial hypertension due to venous outflow obstruction. In this population flow velocity and relative pressure measurement can assist in the monitoring of therapy and assess the results in interventions such as dural sinus stenting. Esfahani et al. assessed five patients with intracranial venous hypertension and dural sinus stenosis [81]. The mean pre-stenotic intravenous pressure measured by endovascular catheter placement was $45.2 \mathrm{~mm}$ $\mathrm{Hg}$ and decreased to $27.4 \mathrm{~mm} \mathrm{Hg}$ following stenting. In these patients, the total jugular flow measured by $4 \mathrm{D}$ Flow MRI increased by $260.2 \mathrm{~mL} / \mathrm{min}$. Analysis of changes in intravenous pressure and 4D flow were highly correlated (Pearson correlation $r=0.926$, Wilcoxon signed rank test $p=0.4$ ) and all patients displayed 
clinical improvement at 6 weeks. The role of 4DFlow MRI may significantly expand in this patient population as post processing methods become more generally available to non-invasively calculate pressure gradients within the dural sinus from the velocity data (Fig. 8).

\section{Alterations in 4DFlow and pulsatility related to dementia} There is increasing evidence that cerebral arteries are often morphologically altered and dysfunctional in Alzheimer's disease (AD) [82]. Cerebral blood flow, arterial pulsation and vasomotion are reduced in $\mathrm{AD}$ patients, and thus the normal perivascular transmission of metabolites out of the brain is diminished. The decrease in perivascular drainage may allow beta-amyloid protein to accumulate within the vessel wall. Vessel rigidity due to arteriosclerosis, atherosclerosis and amyloid deposition will ultimately translate into the inability to dissipate the systolic pressure created by the heart. Consequently, there is interest in non-invasive methods to assess cerebrovascular hemodynamics such as mean blood flow rate and pulsatility index $(\mathrm{PI}=(\mathrm{QmaxQmin}) / \mathrm{Qmean} ; \mathrm{Q}=$ flow $)$ as potential systemic indicators of $\mathrm{AD}$. The $\mathrm{PI}$ is a parameter dependent on the cerebrovascular resistance, the pulse amplitude of arterial pressure, and compliance of the cerebral arterial bed, and thus serves to characterize the cerebrovascular health. Due to temporal averaging, 4Dflow MRI mean velocities are approximately $30 \%$ lower compared with transcranial Doppler ultrasound accounting for the differences between the techniques [83].

4Dflow MRI has demonstrated significant differences in the cerebral hemodynamics of an $\mathrm{AD}$ group when compared to cognitively healthy age matched controls [84]. There is a significant decrease in arterial mean blood flow in the AD population and a significant increase in PI, particularly the middle cerebral arteries and the cavernous internal carotids. An increased in intracranial PI is an indicator of increased distal resistance to blood flow within the microvasculature which is often related to small vessel disease [85]. These results are in agreement with other studies that have used TCD to determine the effect of $\mathrm{AD}$ on blood flow to the brain [82].

\section{Conclusion}

The barriers to the routine use of 4DFlow MRI to study cerebrovascular hemodynamics are gradually being overcome. The combination of parallel imaging, spatiotemporal acceleration and non-Cartesian sampling provides increase spatial resolution ( $<0.7$ isotropic) and whole brain coverage in practical imaging times. Commercial packages are in development which will enable the
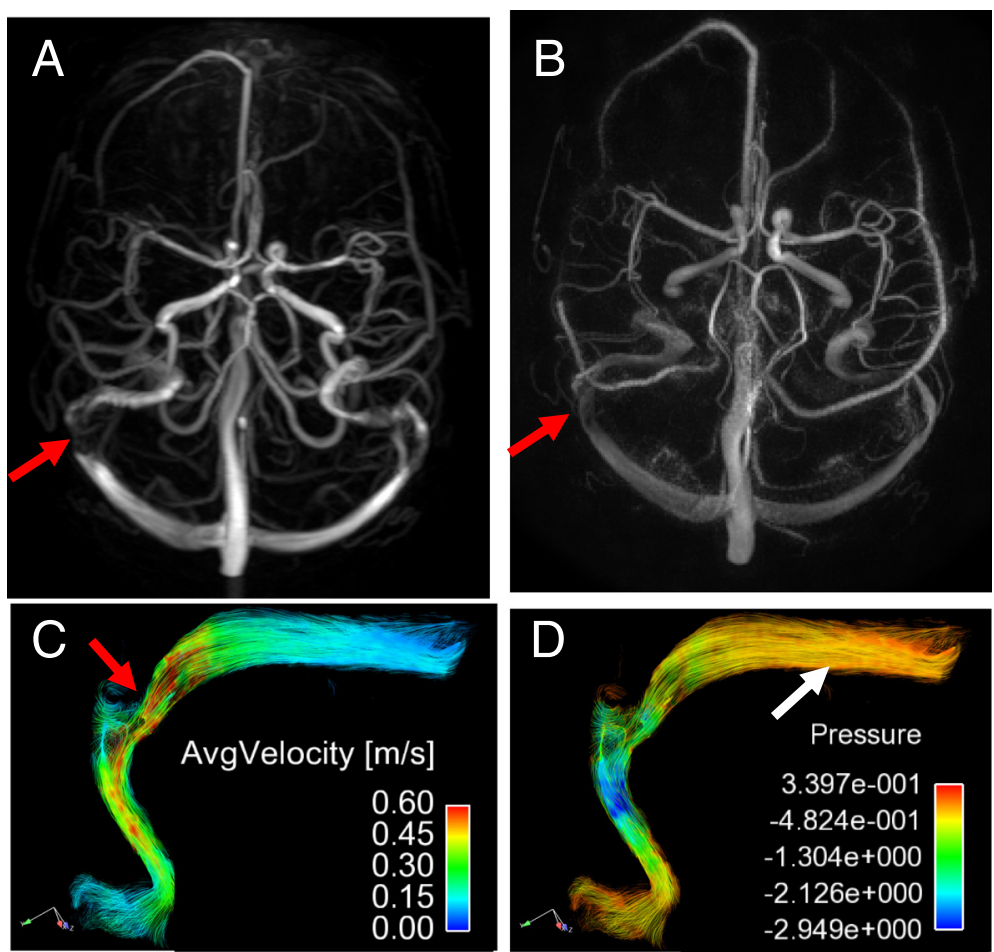

Fig. 8 Idiopathic intracranial hypertension. a The axial 4DFlow MRI image demonstrates a stenosis of the right transverse sinus (arrow). b The CE MRA confirms a stenosis at the junction of the transvers and sigmoid sinus (arrow). c The 4DFlow MRI velocities are elevated in the stenotic segment (arrow). d The relative pressure map reveals higher pressure in the transvers sinus (white arrow) compared to the stenotic segment and distal sigmoid sinus 
clinical implementation of 4Flow and hemodynamic analysis. The physiological parameters derived from 4DFlow MRI will augment anatomical imaging and has the potential to improve characterization of the entire spectrum of cerebrovascular diseases.

\section{Competing interests}

The authors declare that they have no competing interests.

\section{Authors' contributions}

PT concept, data acquisition, manuscript preparation and editing. Address: Radiology, 600 Highland Ave, Madison 53792 Wisconsin USA. AS manuscript preparation. EH manuscript preparation. ZC manuscript preparation. TS data analysis. LR data analysis. YW data analysis. OW concept, design, development, data acquisition. KJ data acquisition, data analysis, design, manuscript preparation and editing. All authors read and approved the final manuscript.

\section{Author details}

'Department of Radiology, University of Wisconsin School of Medicine and Public Health, Madison, Wisconsin, USA. ${ }^{2}$ Department of Medical Physics, University of Wisconsin School of Medicine and Public Health, Madison, Wisconsin, USA. ${ }^{3}$ Department of Clinic of Radiology and Nuclear Medicine, Basel, Switzerland.

\section{Received: 28 February 2016 Accepted: 18 April 2016}

\section{Published online: 22 April 2016}

\section{References}

1. Moran PR. A flow velocity zeugmatographic interlace for NMR imaging in humans. Magn Reson Imaging. 1982;1(4):197-203.

2. Dumoulin CL, Hart Jr HR. Magnetic resonance angiography. Radiology. 1986; 161(3):717-20.

3. Dumoulin $\mathrm{CL}$, Souza SP, Walker MF, Wagle W. Three-dimensional phase contrast angiography. Magn Reson Med. 1989;9(1):139-49.

4. Pelc NJ, Herfkens RJ, Shimakawa A, Enzmann DR. Phase contrast cine magnetic resonance imaging. Magn Reson Q. 1991;7(4):229-54.

5. Wigstrom L, Sjoqvist L, Wranne B. Temporally resolved $3 D$ phase-contrast imaging. Magn Reson Med. 1996;36(5):800-3.

6. Chang W, Huang M, Chien A. Emerging techniques for evaluation of the hemodynamics of intracranial vascular pathology. Neuroradiol J. 2015;28(1):19-27.

7. Bauer S, Markl M, Foll D, et al. K-t GRAPPA accelerated phase contrast MRI: Improved assessment of blood flow and 3-directional myocardial motion during breath-hold. J Magn Reson Imaging. 2013;38(5):1054-62.

8. Bauer S, Markl M, Honal M, Jung BA. The effect of reconstruction and acquisition parameters for GRAPPA-based parallel imaging on the image quality. Magn Reson Med. 2011:66(2):402-9.

9. Stadlbauer A, van der Riet W, Crelier G, Salomonowitz E. Accelerated timeresolved three-dimensional MR velocity mapping of blood flow patterns in the aorta using SENSE and k-t BLAST. Eur J Radiol. 2010;75(1):e15-21.

10. Petersson $S$, Sigfridsson A, Dyverfeldt $P$, et al. Retrospectively gated intracardiac 4D flow MRI using spiral trajectories. Magn Reson Med. 2015;76:196-206.

11. Chang W, Frydrychowicz A, Kecskemeti S, et al. The effect of spatial resolution on wall shear stress measurements acquired using radial phase contrast magnetic resonance angiography in the middle cerebral arteries of healthy volunteers. Preliminary results. Neuroradiol J. 2011;24(1):115-20.

12. Wu Y, Chang W, Johnson KM, et al. Fast whole-brain 4D contrast-enhanced MR angiography with velocity encoding using undersampled radial acquisition and highly constrained projection reconstruction: image-quality assessment in volunteer subjects. AJNR Am J Neuroradiol. 2011;32(3):E47-50.

13. Gu T, Korosec FR, Block WF, et al. PC VIPR: a high-speed 3D phase-contrast method for flow quantification and high-resolution angiography. AJNR Am J Neuroradiol. 2005;26(4):743-9.

14. Johnson KM, Markl M. Improved SNR in phase contrast velocimetry with fivepoint balanced flow encoding. Magn Reson Med. 2010;63(2):349-55.

15. Wetzel S, Meckel S, Frydrychowicz A, et al. In vivo assessment and visualization of intracranial arterial hemodynamics with flow-sensitized 4D MR imaging at 3T. AJNR Am J Neuroradiol. 2007;28(3):433-8.

16. Jung $B$, Honal M, Ullmann $P$, et al. Highly k-t-space-accelerated phasecontrast MRI. Magn Reson Med. 2008;60(5):1169-77.
17. Bock J, Frydrychowicz A, Stalder AF, et al. 4D phase contrast MRI at 3T: effect of standard and blood-pool contrast agents on SNR, PC-MRA, and blood flow visualization. Magn Reson Med. 2010;63(2):330-8.

18. Liu J, Dyverfeldt P, Acevedo-Bolton G, et al. Highly accelerated aortic 4D flow MR imaging with variable-density random undersampling. Magn Reson Imaging. 2014;32(8):1012-20.

19. Schnell S, Markl M, Entezari P, et al. k-t GRAPPA accelerated four-dimensional flow MRI in the aorta: effect on scan time, image quality, and quantification of flow and wall shear stress. Magn Reson Med. 2014;72(2):522-33.

20. Stankovic Z, Allen BD, Garcia J, et al. 4D flow imaging with MRI. Cardiovasc Diagn Ther. 2014;4(2):173-92

21. Callaghan FM, Kozor R, Sherrah AG, et al. Use of multi-velocity encoding 4D flow MRI to improve quantification of flow patterns in the aorta. J Magn Reson Imaging. 2015;42:352-63.

22. Nayak KS, Nielsen JF, Bernstein MA, et al. Cardiovascular magnetic resonance phase contrast imaging. J Cardiovasc Magn Reson. 2015;17(1):71.

23. Hutter J, Schmitt P, Aandal G, et al. Low-rank and sparse matrix decomposition for compressed sensing reconstruction of magnetic resonance $4 \mathrm{D}$ phase contrast blood flow imaging (loSDeCoS 4D-PCI). Med Image Comput Comput Assist Interv. 2013;16(Pt 1):558-65.

24. Boussel L, Rayz V, Martin A, et al. Phase-contrast magnetic resonance imaging measurements in intracranial aneurysms in vivo of flow patterns, velocity fields, and wall shear stress: comparison with computational fluid dynamics. Magn Reson Med. 2009;61(2):409-17.

25. Schubert T, Santini F, Stalder AF, et al. Dampening of blood-flow pulsatility along the carotid siphon: does form follow function? AJNR Am J Neuroradiol. 2011; 32(6):1107-12

26. Kim GB, Ha H, Kweon J, et al. Post-stenotic plug-like jet with a vortex ring demonstrated by 4D flow MRI. Magn Reson Imaging. 2015:34:371-5.

27. Krittian SB, Lamata P, Michler C, et al. A finite-element approach to the direct computation of relative cardiovascular pressure from time-resolved MR velocity data. Med Image Anal. 2012;16(5):1029-37.

28. Bustamante M, Petersson S, Eriksson J, et al. Atlas-based analysis of 4D flow CMR: automated vessel segmentation and flow quantification. J Cardiovasc Magn Reson. 2015;17:87.

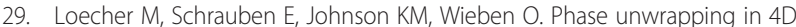
MR flow with a 4D single-step laplacian algorithm. J Magn Reson Imaging. 2015:43:833-42.

30. von Spiczak J, Crelier G, Giese D, et al. Quantitative Analysis of Vortical Blood Flow in the Thoracic Aorta Using 4D Phase Contrast MRI. PLoS One. 2015;10(9):e0139025

31. Santelli C, Loecher M, Busch J, et al. Accelerating 4D flow MRI by exploiting vector field divergence regularization. Magn Reson Med. 2016;75(1):115-25.

32. Frydrychowicz A, Francois CJ, Turski PA. Four-dimensional phase contrast magnetic resonance angiography: potential clinical applications. Eur J Radiol. 2011;80(1):24-35

33. Chimowitz MI, Lynn MJ, Derdeyn CP, et al. Stenting versus aggressive medical therapy for intracranial arterial stenosis. N Engl J Med. 2011 365(11):993-1003.

34. Traub O, Berk BC. Laminar shear stress: mechanisms by which endothelial cells transduce an atheroprotective force. Arterioscler Thromb Vasc Biol. 1998;18(5):677-85.

35. Harloff A, Albrecht F, Spreer J, et al. 3D blood flow characteristics in the carotid artery bifurcation assessed by flow-sensitive 4D MRI at 3T. Magn Reson Med. 2009:61(1):65-74.

36. Markl M, Wegent $F$, Zech $T$, et al. In vivo wall shear stress distribution in the carotid artery: effect of bifurcation geometry, internal carotid artery stenosis, and recanalization therapy. Circ Cardiovasc Imaging. 2010;3(6):647-55

37. Harloff A, Zech T, Wegent F, et al. Comparison of blood flow velocity quantification by 4D flow MR imaging with ultrasound at the carotid bifurcation. AJNR Am J Neuroradiol. 2013;34(7):1407-13.

38. Leng $X$, Scalzo F, Ip HL, et al. Computational fluid dynamics modeling of symptomatic intracranial atherosclerosis may predict risk of stroke recurrence. PLoS One. 2014;9(5):e97531.

39. Zhao X, Zhao M, Amin-Hanjani S, et al. Wall shear stress in major cerebral arteries as a function of age and gender-a study of 301 healthy volunteers. J Neuroimaging. 2015;25(3):403-7.

40. Amin-Hanjani $S$, Pandey DK, Rose-Finnell $L$, et al. Effect of hemodynamics on stroke risk in symptomatic atherosclerotic vertebrobasilar occlusive disease. JAMA Neurol. 2015;73:178-85. 
41. Amin-Hanjani S, Du X, Zhao M, et al. Use of quantitative magnetic resonance angiography to stratify stroke risk in symptomatic vertebrobasilar disease. Stroke. 2005;36(6):1140-5.

42. Bauer AM, Amin-Hanjani S, Alaraj A, Charbel FT. Quantitative magnetic resonance angiography in the evaluation of the subclavian steal syndrome: report of 5 patients. J Neuroimaging. 2009;19(3):250-2.

43. Sekine T, Takagi R, Amano Y, et al. 4D flow MRI assessment of extracranialintracranial bypass: qualitative and quantitative evaluation of the hemodynamics. Neuroradiology. 2015;58:237-44.

44. Kellawan JM, Harrell JW, Schrauben EM, et al. Quantitative cerebrovascular 4D flow MRI at rest and during hypercapnia challenge. Magn Reson Imaging. 2015;34:422-8.

45. Qiao Y, Zeiler SR, Mirbagheri S, et al. Intracranial plaque enhancement in patients with cerebrovascular events on high-spatial-resolution MR images. Radiology. 2014;271(2):534-42.

46. Dieleman N, van der Kolk AG, Zwanenburg JJ, et al. Imaging intracranial vessel wall pathology with magnetic resonance imaging: current prospects and future directions. Circulation. 2014;130(2):192-201.

47. Skarpathiotakis M, Mandell DM, Swartz RH, et al. Intracranial atherosclerotic plaque enhancement in patients with ischemic stroke. AJNR Am J Neuroradiol. 2013;34(2):299-304.

48. Xu WH, Li ML, Gao S, et al. Middle cerebral artery intraplaque hemorrhage: prevalence and clinical relevance. Ann Neurol. 2012;71(2):195-8.

49. Turan TN, Bonilha L, Morgan PS, et al. Intraplaque hemorrhage in symptomatic intracranial atherosclerotic disease. J Neuroimaging. 2011;21(2):e159-61.

50. Xu WH, Li ML, Gao S, et al. In vivo high-resolution MR imaging of symptomatic and asymptomatic middle cerebral artery atherosclerotic stenosis. Atherosclerosis. 2010;212(2):507-11.

51. Sun J, Yuan C. Seeking culprit lesions in cryptogenic stroke: The utility of vessel wall imaging. J Am Heart Assoc. 2015;4(6):002207.

52. Freilinger TM, Schindler A, Schmidt C, et al. Prevalence of nonstenosing, complicated atherosclerotic plaques in cryptogenic stroke. JACC Cardiovasc Imaging. 2012;5(4):397-405.

53. Gupta A, Gialdini G, Lerario MP, et al. Magnetic resonance angiography detection of abnormal carotid artery plaque in patients with cryptogenic stroke. J Am Heart Assoc. 2015;4(6):e002012.

54. Oeltze-Jafra S, Cebral JR, Janiga G, Preim B. Cluster analysis of vortical flow in simulations of cerebral aneurysm hemodynamics. IEEE Trans Vis Comput Graph. 2016;22(1):757-66.

55. Chung B, Cebral JR. CFD for evaluation and treatment planning of aneurysms: review of proposed clinical uses and their challenges. Ann Biomed Eng. 2015;43(1):122-38.

56. Cebral JR, Vazquez M, Sforza DM, et al. Analysis of hemodynamics and wall mechanics at sites of cerebral aneurysm rupture. J Neurointerv Surg. 2015;7(7):530-6.

57. Cebral JR, Duan X, Chung BJ, et al. Wall mechanical properties and hemodynamics of unruptured intracranial aneurysms. AJNR Am J Neuroradiol. 2015;36(9):1695-703.

58. Berg P, Roloff C, Beuing $\mathrm{O}$, et al. The computational fluid dynamics rupture challenge 2013-phase II: Variability of hemodynamic simulations in two intracranial aneurysms. J Biomech Eng. 2015;137(12):121008.

59. Schnell S, Ansari SA, Vakil $P$, et al. Three-dimensional hemodynamics in intracranial aneurysms: influence of size and morphology. J Magn Reson Imaging. 2014;39(1):120-31.

60. Meckel S, Stalder AF, Santini F, et al. In vivo visualization and analysis of 3-D hemodynamics in cerebral aneurysms with flow-sensitized 4-D MR imaging at 3T. Neuroradiology. 2008;50(6):473-84.

61. Isoda $\mathrm{H}$, Hirano M, Takeda $\mathrm{H}$, et al. Visualization of hemodynamics in a silicon aneurysm model using time-resolved, 3D, phase-contrast MRI. AJNR Am J Neuroradiol. 2006;27(5):1119-22.

62. van Ooij P, Schneiders JJ, Marquering HA, et al. 3D cine phase-contrast MRI at $3 \mathrm{~T}$ in intracranial aneurysms compared with patient-specific computational fluid dynamics. AJNR Am J Neuroradiol. 2013;34(9):1785-91.

63. Sforza DM, Kono K, Tateshima S, et al. Hemodynamics in growing and stable cerebral aneurysms. J Neurointerv Surg. 2015;8:407-12.

64. Meng H, Tutino VM, Xiang J, Siddiqui A. High WSS or low WSS? Complex interactions of hemodynamics with intracranial aneurysm initiation, growth, and rupture: toward a unifying hypothesis. AJNR Am J Neuroradiol. 2014; 35(7):1254-62.

65. Matouk CC, Mandell DM, Gunel M, et al. Vessel wall magnetic resonance imaging identifies the site of rupture in patients with multiple intracranial aneurysms: proof of principle. Neurosurgery. 2013;72(3):492-6. discussion 6.
66. Nagahata S, Nagahata M, Obara M, et al. Wall Enhancement of the Intracranial Aneurysms Revealed by Magnetic Resonance Vessel Wall Imaging Using Three-Dimensional Turbo Spin-Echo Sequence with MotionSensitized Driven-Equilibrium: A Sign of Ruptured Aneurysm? Clin Neuroradiol. 2014 [Epub ahead of print].

67. Edjlali M, Gentric JC, Regent-Rodriguez C, et al. Does aneurysmal wall enhancement on vessel wall MRI help to distinguish stable from unstable intracranial aneurysms? Stroke. 2014;45(12):3704-6.

68. Blankena R, Kleinloog R, Verweij BH, et al. Thinner regions of intracranial aneurysm wall correlate with regions of higher wall shear stress: A 7T MRI Study. AJNR Am J Neuroradiol. 2016 [Epub ahead of print].

69. Greving JP, Wermer MJ, Brown Jr RD, et al. Development of the PHASES score for prediction of risk of rupture of intracranial aneurysms: a pooled analysis of six prospective cohort studies. Lancet Neurol. 2014;13(1):59-66.

70. Spetzler RF, Martin NA. A proposed grading system for arteriovenous malformations. J Neurosurg. 1986;65(4):476-83.

71. Chang W, Loecher MW, Wu Y, et al. Hemodynamic changes in patients with arteriovenous malformations assessed using high-resolution 3D radial phasecontrast MR angiography. AJNR Am J Neuroradiol. 2012;33(8):1565-72.

72. Edjlali M, Roca P, Rabrait C, et al. MR selective flow-tracking cartography: a postprocessing procedure applied to four-dimensional flow MR imaging for complete characterization of cranial dural arteriovenous fistulas. Radiology. 2014;270(1):261-8

73. Wu C, Ansari SA, Honarmand AR, et al. Evaluation of $4 D$ vascular flow and tissue perfusion in cerebral arteriovenous malformations: influence of Spetzler-Martin grade, clinical presentation, and AVM risk factors. AJNR Am J Neuroradiol. 2015;36(6):1142-9.

74. Markl M, Wu C, Hurley MC, et al. Cerebral arteriovenous malformation: complex 3D hemodynamics and 3D blood flow alterations during staged embolization. J Magn Reson Imaging. 2013;38(4):946-50.

75. Wu C, Schnell S, Markl M, Ansari SA. Combined DSA and 4D flow demonstrate overt alterations of vascular geometry and hemodynamics in an unusually complex cerebral AVM. Clin Neuroradiol. 2015 [Epub ahead of print].

76. Cognard C, Gobin YP, Pierot L, et al. Cerebral dural arteriovenous fistulas: clinical and angiographic correlation with a revised classification of venous drainage. Radiology. 1995;194(3):671-80.

77. Chang W, Wu Y, Johnson $K$, et al. Fast contrast-enhanced 4D MRA and 4D flow MRI using constrained reconstruction (HYPRFlow): potential applications for brain arteriovenous malformations. AJNR Am J Neuroradiol. 2015;36(6):1049-55.

78. Clark Z, Johnson KM, Wu Y, et al. Accelerated time-resolved contrastenhanced magnetic resonance angiography of dural arteriovenous fistulas using highly constrained reconstruction of sparse cerebrovascular data sets. Invest Radiol. 2015 [Epub ahead of print].

79. Schrauben EM, Johnson KM, Huston J, et al. Reproducibility of cerebrospinal venous blood flow and vessel anatomy with the use of phase contrastvastly undersampled isotropic projection reconstruction and contrastenhanced MRA. AJNR Am J Neuroradiol. 2014;35(5):999-1006.

80. Sari S, Verim S, Hamcan S, et al. MRI diagnosis of dural sinus - Cortical venous thrombosis: Immediate post-contrast 3D GRE T1-weighted imaging versus unenhanced MR venography and conventional MR sequences. Clin Neurol Neurosurg. 2015;134:44-54.

81. Esfahani DR, Stevenson M, Moss HE, et al. Quantitative Magnetic Resonance Venography is Correlated With Intravenous Pressures Before and After Venous Sinus Stenting: Implications for Treatment and Monitoring. Neurosurgery. 2015;77(2):254-60.

82. Roher AE. Cardiovascular system participation in Alzheimer's disease pathogenesis. J Intern Med. 2015;277(4):426-8.

83. Chang $W$, Landgraf $B$, Johnson KM, et al. Velocity measurements in the middle cerebral arteries of healthy volunteers using 3D radial phase-contrast HYPRFlow: comparison with transcranial Doppler sonography and 2D phase-contrast MR imaging. AJNR Am J Neuroradiol. 2011:32(1):54-9.

84. Rivera-Rivera LA, Turski $P$, Johnson KM, et al. 4D flow MRI for intracranial hemodynamics assessment in Alzheimer's disease. J Cereb Blood Flow Metab. 2015 [Epub ahead of print].

85. Peng X, Haldar S, Deshpande S, et al. Wall stiffness suppresses Akt/eNOS and cytoprotection in pulse-perfused endothelium. Hypertension. 2003; 41(2):378-81. 